\title{
Premenstrual Syndrome (PMS) and the Myth of the Irrational Female
}

\author{
Sally King
}

Ever since 'premenstrual' symptoms were first formally described in 1931, those most prominently cited in diagnostic criteria have been mood-based (Laws, Hey, and Eagan 1985; Ussher 2011). However, the reason for prioritizing psychological symptoms over physical ones remains unclear. For example, population studies have shown that they are not necessarily the most commonly experienced, uniquely determining, nor most disruptive of menstrual changes (for example, Dennerstein, Lehert, and Heinemann 2011; Choi et al. 2010). This chapter argues that this trend is influenced by a sexist historical assumption, referred to here as 'the myth of the irrational female'; the idea that women are pathologically emotional, and thus have a reduced capacity for reason, due to their reproductive biology.

I begin by outlining some historical shifts in medical descriptions of female-prevalent symptoms, to help explain why cyclical mood changes came to be prioritized over physical experiences, such as pain. I then use the latest Royal College of Obstetricians and Gynaecologists guidelines on PMS (RCOG 2016) to show that although clinical experts are trying to overcome this issue, they have not yet managed to completely disentangle PMS from the myth of the irrational female. I conclude by calling for a more integrated and rigorous approach to PMS definitions and research, in order to support people who do experience cyclical symptoms, without unintentionally pathologizing the menstrual cycle, or stigmatizing an entire gender. 


\section{The Psychologizing of Menstrual Cycle-Related Symptoms}

\section{Women, Wombs, Health, and Reason}

Menstrual cycle-related symptoms have been recognized and treated by physicians for at least the past 3800 years. We know this because one of the oldest surviving medical texts, the Kahun Gynaecological Papyrus (c. $1800 \mathrm{BCE}$ ), describes some highly recognizable menstrual experiences (for example, period pain, heavy bleeding, lower back pain, and migraine) and ascribes them to 'discharges . . clenches . . wrappings . . or wandering ... of the womb' (Quirke 2002, 1:1-29). Interestingly, this Ancient Egyptian scroll also lists other (less directly associated) female symptoms and similarly associates them with the womb for example, leg pain and toothache (Quirke 2002, $1: 15-20)$.

In this way, the Kabun Gynaecological Papyrus perhaps exemplifies a recurring theme that has helped shape constructions of female health throughout the history of Western medicine, namely, the attribution of any and all (otherwise unexplained) female-prevalent symptoms to the womb (or menstruation by association), as the defining feature of embodied femininity (Ussher 1989; King 1998). While a causal association between the female reproductive system and ill health is not necessarily 'wrong' or sexist, a widespread (yet incorrect) assumption that a generalized converse logical position also holds true, is much more problematic. That is, 'if ill health is caused by the womb (in some women), then all women are ill (by virtue of having wombs).' Indeed, it is this logical fallacy that underpins much of the myth of the irrational female.

Right up until the sixteenth century, descriptions of female-prevalent symptoms were always physical in nature (King 1998). From the seventeenth century onwards, however, they came to be seen as more emotional in origin, and experience. In particular, the work of Thomas Sydenham (1624-1689) was influential in the reclassification of 'hysteria' (a term adapted from Classical references to hysterike pnix that is, 'suffocation of the womb') from a gynecological condition, to a female-prevalent nervous condition, caused by a weaker nervous constitution and subsequent emotional instability (Gilman et al. 1993, 143-46).

Sydenham's new definition of 'hysteria' was in keeping with preexisting philosophical assumptions that women were inherently physically, spiritually, and intellectually inferior to men. Yet it meant that gender discrimination was thus now justified by a 'scientific' (that is, supposedly objective) medical claim that due to the pathological nature of the female (reproductive) body, women were also pathologically emotional (and, therefore, less capable of reason) and so inevitably subject to the control of men (Ussher 2005, 16).

By the late nineteenth century, the work of Sigmund Freud had firmly repositioned 'hysteria' as a type of psychological neurosis (Freud 1966). His work significantly influenced early twentieth century public discourse. 
Indeed, one of the main arguments used against women's suffrage (the right to vote in parliamentary elections) was that (all) women were mentally unfit to make an informed and rational decision. Just such a belief was unambiguously conveyed by Sir Almroth Wright (1912), a famous physiologist and anti-suffragist; "[On] Militant Hysteria- No doctor. . . can ever lose sight of the fact that the mind of woman is always threatened with danger from the reverberations of her physiological emergencies."

\section{The Emergence of Premenstrual Conditions}

The 'myth of the irrational female' is also a likely influence on the first formal medical description of 'Premenstrual Tension' ('PMT'- the precursor to 'PMS') by Frank (1931), a US gynecologist. For although Frank's paper initially documented cases of severe cyclical asthma, epilepsy, water retention, and cardiac irregularity, its main focus was on the experiences of a specific subset of patients, characterized by various signs of 'nervous tension' (Frank 1931, 1054). Astonishingly, in several cases, Frank's symptom descriptions were simply value judgements on improper, or undesired, female behavior; and strikingly similar to contemporary descriptions of the 'hysterical woman' for example, "husband to be pitied," "unbearable, shrew," or "impossible to live with" (Frank 1931, 1055).

Frank was certainly not the first in the modern era to attribute changes in mood or emotions to the menstrual cycle. Various earlier sources describe apparently well-known expressions of cyclical emotional variability in a minority of menstruating women, although they were not previously considered to require medical intervention (for example, Hollick 1860, 91-92; Giles 1901, 27). Emily Martin $(1987,120)$ suggests that it is highly significant that Frank specifically discussed PMT in relation to women's (supposed lesser) ability to work, since he was writing during the Great Depression, a time when women workers were being pressured to leave paid employment in favor of men.

Also, the first estrogen hormone was discovered just three years before Frank published his paper. The identification of the so-called 'sex hormones' provided a new scientific explanation to support existing ideas about the female reproductive system as the origin of female-prevalent ill health, and implied 'natural' susceptibility to irrational behaviors (Ussher 2011, 21). Correspondingly, Frank associated such overtly feminine symptoms (that is, those affecting behavior, mood, or emotions) with an excess of the 'female' hormone (now disproved) (Frank 1931, 1056):

It would thus appear that the continued circulation of an excessive amount of female sex hormone in the blood may in labile persons produce serious symptoms, some cardiovascular, but the most striking definitely psychic and nervous (autonomic). These periodic attacks ... can be directly ascribed to the excessive hormonal stimulus. 
During the 1950s, theories about Premenstrual Tension (PMT) continued to focus on the role of 'female sex hormones,' although symptoms were now attributed to a deficiency in progesterone (now disproved). PMT was renamed PMS (Premenstrual Syndrome), after Greene and Dalton (1953) argued that premenstrual symptoms were far more extensive than just 'nervous tension.' The most prominent 'PMS expert' at this time, Dr. Katharina Dalton, attempted to counter the undue emphasis on mood-based menstrual symptoms, and openly criticized what she called "the hijacking of PMS by psychologists" (Dalton and Holton 2000, 98). However, she also helped to perpetuate the myth of the irrational female by directly contributing to a somewhat dangerous (and unscientific) legal precedent for the mitigation of murder charges, if committed 'under the influence of PMS' (Laws, Hey, and Eagan 1985, 65-79; Chrisler 2002).

Over the past thirty years, clinical descriptions of PMS have remained predominantly psychological in focus, especially since the inclusion of premenstrual disorders in the American Psychiatric Association's DSM (Diagnostic and Statistical Manual of mental disorders). First in the form of Late Luteal Phase Dysphoric Disorder (LLPDD) in 1987, and later its replacement, Premenstrual Dysphoric Disorder (PMDD), since 1994 (American Psychiatric Association 2000). Even reputable clinical sources sometimes refer to PMDD as 'severe PMS'; implying that PMS is simply a less severe form of a mental health disorder (for example, Lopez, Kaptein, and Helmerhorst 2012; Maharaj and Trevino 2015; Naheed et al. 2017). In comparison, thyroid conditions, which are also 'hormonal' in origin and commonly cause severe mood changes, are not listed in the DSM.

\section{So What? The Impact of Psychologising PMS}

PMS research has, so far, been typified by contradictory, irreplicable, and usually highly contested, findings (Walker 1997; Knaapen and Weisz 2008; Halbreich 2007). This may partly be explained by the fact that for most of its history, it has been subject to a form of confirmation bias, or circular logic. By focussing on mood-based menstrual symptoms and neglecting those that are physical, PMS research unavoidably overlooks critical elements in the etiology (cause), prevalence, patient experiences, and treatments of menstrual cycle-related symptoms as a whole. For example, period pain is by far the most common menstrual cycle-related symptom, but it is not usually included in diagnostic criteria for PMS. This is despite the fact that period pain is known to have an effect on premenstrual mood, fatigue, and other symptoms (Balik et al. 2014; Smorgick et al. 2013).

Clinicians may struggle to identify menstrual cycle-related symptoms if PMS is understood to be essentially mood-based. For example, if certain physical symptoms are severe, a General Practitioner (GP) might simply diagnose a more chronic health condition, without first assessing if symptoms are cyclical in nature. Especially since even when PMS is suspected, many 
clinicians do not ask patients to record their symptoms over two cycles, as is required for formal diagnosis (Craner, Sigmon, and McGillicuddy 2014). (Menstrual migraine is an exception, since its high prevalence rate ensures that most clinicians are aware of a possible link to the menstrual cycle.)

Some clinicians may even question the validity of a PMS diagnosis, partly because symptoms are subjective and, therefore, difficult to qualify (for example, through clinical tests), but also because female-prevalent health issues in general, especially those that also affect mood (for example, fibromyalgia, chronic fatigue syndrome, irritable bowel syndrome [IBS]), are more likely to be dismissed, or assumed to be psychosomatic in origin (see Hoffmann and Tarzian 2001; Asbring and Närvänen 2002; Letson and Dancey 1996). This troubling situation is also likely to be influenced by the myth of the irrational female, since hypochondria (imagined or pretend illness) was emphasized in nineteenth century descriptions of 'female hysteria' (Veith 1965, 144-45).

Systematic reviews of published clinical trials are felt to be the 'gold standard for clarity, power, and precision' in regard to the evidence-based management of PMS (O'Brien and Ismail 2007, 6). However, all five of the existing Cochrane systematic reviews of PMS treatments are based on clinical trials that almost universally selected participants using predominantly mood-based criteria (Jing et al. 2009; Lopez, Kaptein, and Helmerhorst 2012; Ford et al. 2012; Marjoribanks et al. 2013; Naheed et al. 2017). This is because the most widely used clinical tools for recording daily symptoms are either directly based on the diagnostic criteria for PMDD for example, the Daily Record of Severity of Problems (DRSP) (Endicott, Nee, and Harrison 2006), or otherwise over-emphasize emotional, behavioral, or psychological symptoms for example, variations of the Moos Menstrual Distress Questionnaire (MDQ) (Moos 1968). So, in effect, only a small subset of PMS patients/ symptoms have ever been evaluated in the vast majority of clinical trials.

Some PMS researchers have focused on identifying cultural influences on clinical and scientific definitions of PMS. Their work is sometimes also limited by the assumption that menstrual changes are predominantly mood-based. For example, arguments put forward to oppose the unnecessary 'medicalization' of the menstrual cycle (while acknowledging the reality of the experiences of those who do experience distressing cyclical mood changes), may downplay, or neglect to mention physical menstrual cycle-related symptoms or conditions such as period pain, catamenial epilepsy, menstrual migraine, or cyclical asthma (for example, Chrisler and Gorman 2015). This omission might be seen to undermine their arguments (Kulkarni 2013), even though the point is still valid: The fact that a minority of people experience symptoms that are triggered by the menstrual cycle, does not justify the pathologisation of the cycle itself.

Research has found that people are more likely to report PMS if they have restricted access to social support (for example, Ussher, Perz, and Mooney-Somers 2007); an unequal share of household or childrearing responsibilities (for example, Coughlin 1990; Ussher 2003); or are experiencing 
relationship strain (for example, Kuczmierczyk, Labrum, and Johnson 1992). Any mention of 'psychosomatic,' or 'psycho-social' factors in relation to PMS, however, can trigger defensiveness on the part of the patient, their clinicians, or the wider public. Such terms are erroneously equated with hypochondria, even though this is not what is being described. Sadly, the resulting defensiveness can lead to the misinterpretation of clinical research findings. For example, a high-quality review that found inconclusive proof to support the existence of a 'specific premenstrual negative mood syndrome' in the general menstruating population (Romans et al. 2012) was misunderstood by some as denying the existence of severe cyclical mood symptoms, entirely (for example, Kulkarni 2013).

\section{Redefining PMS: Where Are We Now?}

The latest RCOG practitioner guidelines are a good representation of the current clinical definition of PMS (RCOG 2016). While it is clear that the guidelines are trying to move on from psychologised models of PMS, they also reveal the difficulty of doing so, especially within the parameters set by Evidence-Based Medicine (EBM) that is, ensuring that recommendations are supported by high-quality empirical evidence (Knaapen and Weisz 2008). Therefore, a close analysis of the guidelines is a useful exercise in identifying anything that could be hindering their application in clinical practice, or unintentionally reinforcing the myth of the irrational female.

To start with, the wording of the RCOG definition inadvertently evokes earlier descriptions of PMS (for example, 'a vast array of psychological symptoms ...') but otherwise takes extreme care to highlight the wide range of potential PMS symptoms, and the fact that it is their timing, and level of severity that indicate a legitimate diagnosis (RCOG 2016, 80):

PMS encompasses a vast array of psychological symptoms such as depression, anxiety, irritability, loss of confidence and mood swings. There are also physical symptoms, typically bloatedness [sic] and mastalgia [breast pain]. It is the timing, rather than the types of symptoms, and the degree of impact on daily activity that supports a diagnosis of PMS. The character of symptoms in an individual patient does not influence the diagnosis ...

This seemingly simple definition is still not straightforwardly applied in the clinical context, however, or entirely free from evoking the myth of the irrational female.

\section{Timing Is Everything}

The term 'premenstrual' actually refers to the entire luteal phase of the menstrual cycle. This phase is typically 14 days long; beginning at ovulation and finishing when menstruation begins (Sonntag and Ludwig 2012). According 
to the RCOG guidelines, in order to qualify as PMS, symptoms “...must be present during the luteal phase and abate as menstruation begins... then [be] followed by a symptom-free week" (RCOG 2016, 80). However, cyclical symptoms often continue beyond the luteal phase, 2-4 days into menstruation (Hartlage et al. 2012). Also, as is typical of PMS research in general, this definition is based on an assumed 28-day menstrual cycle, which is merely an average cycle length. The 'normal' range of menstrual cycle length is usually cited as being anywhere between 21 and 35 days (for example, Simon et al. 2014,702 ). This means that it may be impossible for someone with a 21 -day cycle to ever experience a 'symptom-free' week, since they have only just finished menstruating when they are once again in the luteal phase of their cycle.

Also, by limiting the timing of symptoms to the luteal phase, those most closely associated with menstruation (for example, period pain, fatigue, lower back pain, other muscle/joint pain, or upset digestion) are potentially excluded from the otherwise apparently limitless list of possible menstrual cycle-related symptoms. As discussed in greater detail below, while this may seem clinically 'useful' on a superficial level (in terms of differentiating PMS from other more chronic health conditions), it also contributes to the somewhat arbitrary separation of undeniably physiological cyclical changes from those that are positioned as somehow more psychological in nature, despite their shared origin (Halbreich 2007, 13).

\section{Spot the Difference-Normal Menstrual Change or PMS Symptom?}

Differentiating 'normal menstrual changes,' or 'premenstrually exacerbated conditions,' from 'PMS symptoms' is perhaps the greatest problem affecting research on this topic (O'Brien 2007, xi). There is no clinical test, or known biomarker, that can be used to diagnose PMS, since the exact biological triggers of premenstrual symptoms remain uncertain, and they are nearly all subjective in nature, relying on patient description rather than any objective measurement of severity (Halbreich 2007, 17). Typically, patients are obliged to track daily menstrual changes over at least two cycles, usually involving an indication of symptom severity, before a doctor can formally diagnose PMS (RCOG 2016, 82).

Since the late 1990s, various attempts have been made to try to make symptom tracking more 'objective'; for example, by applying scores to individual symptoms and then having a total score diagnostic cut off point, or measuring the degree of change in severity over different menstrual phases (Steiner et al. 2011). However, the 'total score' approach can be misleading, especially if a patient experiences relatively few, but severe symptoms; and the 'degree of change' approach assumes linear changes in severity (that is, that changes get progressively worse toward menstruation), which is not reflective of many patient experiences (Halbreich 2007, 17).

This is why the RCOG guidelines provide activity-based criteria regarding symptom severity instead; “... symptoms must be severe enough to affect daily 
functioning" (RCOG 2016, 80). While this is certainly a useful and practical response to the problem of symptom subjectivity, issues remain. For example, even 'normal menstrual changes,' or relatively mild experiences of symptoms such as nausea, period pain, backache, or fatigue, can still affect daily functioning. Secondly, this approach still relies entirely on the quality of the daily rating tool, especially in regard to which symptoms define PMS, and how to quantify subjective experiences. Additionally, by not specifying any common symptoms, some that are severe in nature may be diagnosed as separate conditions, such as migraine, anxiety, or IBS, rather than possible PMS.

This approach also implies that all mild menstrual changes are 'subclinical symptoms,' that is, necessarily negative and pathological phenomena. This is an example of the medicalization of the menstrual cycle, a position that ignores any positive menstrual changes for example, higher energy levels, joyful moods, or increased creativity, and obscures the fact that it is a normal and healthy experience for the vast majority of menstruating people (Chrisler et al. 1994). It also makes value judgments on supposedly feminine attributes such as emotional sensitivity (Caplan, McCurdy-Myers, and Gans 1992; Ussher 2005, 2011; Chrisler and Gorman 2015), which could equally be positioned as a sign of healthy human behavior, given that failure to recognize or express emotions can result in serious health consequences such as suicidal or violent behavior (for example, Berke et al. 2016).

\section{What Counts as a PMS Symptom?}

Nearly all medical conditions are associated with a list of their most common or deterministic symptoms in order to aid diagnosis. However, such a list has always been difficult to establish for PMS (Halbreich 2007, 9). Without a shortlist of the most common experiences, any and every change in physical, psychological, or emotional state, becomes a potential 'symptom' of PMS. As a result, the number of supposedly distinct PMS symptoms has been highly exaggerated. This may be through the inclusion of non-pathological changes such as "loss of confidence" (for example, RCOG 2016, 80), value judgments on stereotypically 'feminine' behaviors such as "nagging" or "act spiteful" (for example, Halbreich et al. 1982, 48-49), or by vaguely referring to over 150 (for example, Moos 1968) or even 200 (for example, Dickerson et al. 2003, 1743) non-listed, but apparently 'previously associated' symptoms.

This situation also makes identifying the population prevalence, causal mechanism(s), or best approaches for the treatment of PMS, extremely technically challenging. For instance, it is impossible to meaningfully compare, or synthesize, population studies that have used different symptom criteria; or to isolate the most likely causes of, or treatments for, cyclical symptoms if they are so numerous, or varied, to lack common analytical properties (Halbreich 2007, 13). The highly individualized, subjective, and biopsychosocial (biological, 
psychological, and social) experience of menstrual cycle-related symptoms also makes them hard to measure, and difficult to control for, in the clinical research environment (Halbreich 2007, 17).

\section{The Priming Effect of PMS Tracking Tools}

Despite all of this confusion, some PMS symptoms really do still count more than others. In practice, it comes down to whichever PMS symptom tracking tool is used by the patient. In the case of the RCOG guidelines (RCOG 2016, 82), there is a clear steer toward promoting the most widely used tracking tools; the DRSP (Endicott, Nee, and Harrison 2006) or the Premenstrual Symptoms Screening Tool (PSST) (Steiner, Macdougall, and Brown 2003). Unfortunately, both of these tools are grounded in the specific diagnostic criteria for PMDD (APA 2000). As a result, emotional and psychological symptoms are over-represented across several supposedly distinct categories, and the majority of (the far more commonly experienced) physical menstrual changes are conflated into just one box (O'Brien and Ismail 2007, 5) (Table 23.1).

Research shows that experiences of PMS are highly susceptible to the 'priming effect' in which prior knowledge of what PMS is, and how it is viewed by wider society, plays a significant role in symptom perception (for example, Marván and Escobedo 1999). This creates an obvious problem for PMS tracking tools, which provide primes for such symptoms instead of open-ended symptom reporting. Indeed, Joan Chrisler has persuasively argued that PMS is a highly 'culture-bound' phenomenon, meaning that it is subject to a particular set of cultural beliefs and practices, rather than being a tangible medical 'truth' that is universally recognized, across all cultures (Chrisler 2012, 165):

\footnotetext{
Data collected from women in Hong Kong... Taiwan .... and mainland China ... indicate that the most commonly reported premenstrual changes are fatigue, water retention, muscular tension, pain, and increased sensitivity to cold. Women in the United States do not report cold sensitivity, and women in China rarely report negative affect [low mood]. The results of these studies support the idea that culture shapes which variations in mood and physical sensations are noticed and which cause concern.
}

As if to prove the point, a few years after Chrisler's piece, an article assessing the reliability and validity of a directly translated version of the DRSP tool among Chinese women was published (Wu et al. 2013). It found that 126 Chinese women reported exactly the same symptoms as those listed on this Western (and PMDD-based) tracking tool. Since it is highly unlikely that Chinese women suddenly started experiencing completely new premenstrual symptoms, it seems reasonable to suggest that the DRSP tool itself predicted which type of symptoms were noticed, and now reported. This priming effect 
Table 23.1 The most widely used PMS symptom tracking tools the 'DRSP' and 'PSST'

\begin{tabular}{|c|c|}
\hline DRSP & PSST \\
\hline $\begin{array}{l}\text { 1. Felt depressed, sad, "down" or "blue," or felt } \\
\text { hopeless; or felt worthless or guilty }\end{array}$ & 1. Anger/irritability \\
\hline 2. Felt anxious, tense, "keyed up," or "on edge." & 2. Anxiety/tension \\
\hline $\begin{array}{l}\text { 3. Had mood swings (i.e., suddenly feeling sad } \\
\text { or tearful) or was sensitive to rejection or feelings } \\
\text { were easily hurt }\end{array}$ & 3. Tearful/increased sensitivity to rejection \\
\hline 4. Felt angry or irritable & 4. Depressed mood/hopelessness \\
\hline $\begin{array}{l}\text { 5. Had less interest in usual activities (work, } \\
\text { school, friends, and hobbies) }\end{array}$ & 5. Decreased interest in work activities \\
\hline 6. Had difficulty concentrating & 6. Decreased interest in home activities \\
\hline $\begin{array}{l}\text { 7. Felt lethargic, tired or fatigued; or had lack } \\
\text { of energy }\end{array}$ & 7. Decreased interest in social activities \\
\hline $\begin{array}{l}\text { 8. Had increased appetite or overate; or had } \\
\text { cravings for specific foods }\end{array}$ & 8. Difficulty concentrating \\
\hline $\begin{array}{l}\text { 9. Slept more, took naps, found it hard to get } \\
\text { up when intended; or had trouble getting to } \\
\text { sleep or staying asleep }\end{array}$ & 9. Fatigue/lack of energy \\
\hline $\begin{array}{l}\text { 10. Felt overwhelmed or unable to cope; or felt } \\
\text { out of control }\end{array}$ & 10. Overeating/food cravings \\
\hline \multirow{5}{*}{$\begin{array}{l}\text { 11. Had breast tenderness, breast swelling, } \\
\text { bloated sensation, weight gain, headache, joint } \\
\text { or muscle pain, or other physical symptoms }\end{array}$} & 11. Insomnia \\
\hline & 12. Hypersomnia (needing more sleep) \\
\hline & 13. Feeling overwhelmed or out of control \\
\hline & 14. Physical symptoms: breast tenderness, \\
\hline & $\begin{array}{l}\text { headaches, joint/muscle pain, bloating, and } \\
\text { weight gain }\end{array}$ \\
\hline
\end{tabular}

Credit: Sally King

of symptom tracking tools is therefore a major source of confirmation bias within PMS research, and one that sadly contributes to contradictory research findings, while reinforcing the myth of the irrational female.

\section{Where Do We Go from Here?}

I would argue that if we are really serious about implementing EBM, then we need to significantly improve the rigor of PMS research methods and tools. Only then might we avoid the confirmation bias created by historical assumptions insisting that cyclical symptoms, as experienced by a minority of menstruating people, are somehow more psychological than physical in origin and experience, or evidence of an inherently pathological female reproductive system. Based on the analysis of the RCOG guidelines above, there are a few issues that could be easily resolved with greater linguistic and logical precision, and the integration of knowledge from 'biopsychosocial' PMS research. 
Firstly, in regard to the timing and naming of cyclical symptoms, the term 'premenstrual syndrome' could be changed to 'peri-menstrual symptoms.' This would make explicit the fact that cyclical symptoms are not technically a 'syndrome' and do not only occur 'just before' menstruation. This would also formally recognize, the role of period pain, by far the most common cyclical symptom, which is known to interact with, if not cause, several others.

Secondly, to help differentiate 'normal menstrual changes' from 'cyclical symptoms,' we could develop an indicative list of the most common cyclical symptoms. This list would exclude any that are: value judgments on femininity, normal human behaviors, duplications, unlikely to ever require medical intervention, or not supported by (unbiased) epidemiological data. This list could then inform symptom tracking tools, and the selection criteria used in clinical trials. As things stand, by stating that "there is no limit on the type or number of symptoms experienced" (RCOG 2016, 80), the RCOG guidelines imply that PMS is somehow 'different' to other illnesses, and unintentionally reinforces the notion that the female (reproductive) body is inherently pathological.

Thirdly, we could integrate known (that is, evidence-based) contributing factors to PMS into symptom tracking tools and clinical guidelines. For example, by including space to note any social, dietary, or lifestyle 'triggers' of symptoms on tracking tools, and by ensuring that clinical guidelines endorse the identification of any such factors and describe a range of treatment options through which they might best be managed. Of course, this integration would need to be communicated in a way that does not accidentally imply that this is a gender-specific phenomenon, or one akin to hypochondria. We could do so by sharing examples of other, well-known, psycho-social factors in ill health, such as nausea or vomiting when feeling nervous, or irritability when feeling hungry or tired.

Finally, we could all consistently remind our clinical and public audiences that only a minority of people who menstruate experience severe cyclical symptoms requiring medical support, and that it does not follow that the menstrual cycle is itself a form of illness, or proof of any 'natural' inferiority of women. This includes: never overstating the implications of research findings, never saying 'women' or 'people who menstruate' when actually meaning 'people who experience PMS,' never presenting PMS as a mood disorder, and no longer selecting 'PMS' research participants or diagnosing 'PMS' using the diagnostic criteria for 'PMDD.'

Explicitly recognizing and countering the myth of the irrational female and its influence on the way in which PMS is clinically described and managed, is an important step toward better supporting those who do experience cyclical symptoms, without unintentionally implying that the menstrual cycle is itself a form of illness, or any sort of 'biological' justification for gender inequality. 


\section{REFERENCES}

American Psychiatric Association. 2000. DSM-IV TR. Diagnostic and Statistical Manual of Mental Disorders. 4th edition TR.

Asbring, Pia, and Anna-liisa Närvänen. 2002. "Women's Experiences of Stigma in Relation to Chronic Fatigue Syndrome and Fibromyalgia." Qualitative Health Research 12 (2): 148-60. https://doi.org/10.1177/104973230201200202.

Balik, Gülşah, Işik Üstüner, Mehmet Kağitci, and Figen Kir Şahin. 2014. "Is There a Relationship between Mood Disorders and Dysmenorrhea?" Journal of Pediatric and Adolescent Gynecology 27 (6): 371-74. https://doi.org/10.1016/j. jpag.2014.01.108.

Berke, Danielle S., Dennis E. Reidy, Brittany Gentile, and Amos Zeichner. 2016. "Masculine Discrepancy Stress, Emotion-Regulation Difficulties, and Intimate Partner Violence.” Journal of Interpersonal Violence, May. https://doi.org/10.1177/ 0886260516650967.

Caplan, Paula J., Joan McCurdy-Myers, and Maureen Gans. 1992. "Should 'Premenstrual Syndrome' Be Called a Psychiatric Abnormality?” Feminism \& Psychology 2 (1): 27-44. https://doi.org/10.1177/0959353592021003.

Choi, Dooseok, Dong Yun Lee, Philippe Lehert, Im Soon Lee, Seok Hyun Kim, and Lorraine Dennerstein. 2010. “The Impact of Premenstrual Symptoms on Activities of Daily Life in Korean Women.” Journal of Psychosomatic Obstetrics and Gynecology. https://doi.org/10.3109/01674820903573920.

Chrisler, Joan C. 2002. "Hormone Hostages: The Cultural Legacy of PMS as a Legal Defense." In Charting a New Course for Feminist Psychology, edited by Lynn H. Collins, Michelle R. Dunlap, and Joan C. Chrisler, 238-52. London: Praeger.

- 2012. "PMS as a Culture-Bound Syndrome." In Lectures on the Psychology of Women, edited by J. C. Chrisler, C. Golden, and P. D. Rozee, 4th ed., 155-71. Long Grove: Waveland Press.

Chrisler, Joan C., Ingrid K. Johnston, Nicole M. Champagne, and Kathleen E. Preston. 1994. "Menstrual Joy: The Construct and Its Consequences." Psychology of Women Quarterly 18 (3): 375-87. https://doi.org/10.1111/ j.1471-6402.1994.tb00461.x.

Chrisler, Joan C., and Jennifer A. Gorman. 2015. "The Medicalization of Women's Moods: Premenstrual Syndrome and Premenstrual Dysphoric Disorder." In The Wrong Prescription for Women: How Medicine and Media Create a 'need' for Treatments, Drugs, and Surgery, 77-98. Santa Barbara, CA: Praeger.

Coughlin, Patricia C. 1990. "Premenstrual Syndrome: How Marital Satisfaction and Role Choice Affect Symptom Severity." Social Work (United States) 35 (4): 351-55. https://doi.org/10.1093/sw/35.4.351.

Craner, Julia R., Sandra T. Sigmon, and Morgan L. McGillicuddy. 2014. "Does a Disconnect Occur between Research and Practice for Premenstrual Dysphoric Disorder (PMDD) Diagnostic Procedures?" Women and Health 54 (3): 232-44. https://doi.org/10.1080/03630242.2014.883658.

Dalton, Katharina, and Wendy M. Holton. 2000. The PMS Bible: The Guide to Understanding and Treating PMS. 6th ed. London: Vermilion.

Dennerstein, Lorraine, Philippe Lehert, and Klaas Heinemann. 2011. "Global Study of Women's Experiences of Premenstrual Symptoms and Their Effects on Daily Life." Menopause International 17 (3): 88-95. https://doi.org/10.1258/ mi.2011.011027. 
Dickerson, Lori M., Pamela J. Mazyk, and Melissa H. Hunter. 2003. "Premenstrual Syndrome." American Family Physician 68 (6): 1743-52.

Endicott, Jean, John Nee, and W. Harrison. 2006. "Daily Record of Severity of Problems (DRSP): Reliability and Validity." Archives of Women's Mental Health 9 (1): 41-49. https://doi.org/10.1007/s00737-005-0103-y.

Ford, Olive, Anne Lethaby, Helen Roberts, and Ben W. J. Mol. 2012. "Progesterone for Premenstrual Syndrome." Cochrane Database of Systematic Reviews. March. https://doi.org/10.1002/14651858.CD003415.pub4.

Frank, Robert T. 1931. "The Hormonal Causes of Premenstrual Tension." Archives of Neurology Psychiatry 26: 1053-57.

Freud, Sigmund. 1966. "Hysteria." In The Standard Edition of the Complete Psychological Works of Sigmund Freud, Volume I (1886-1899): Pre-Psycho-Analytic Publications and Unpublished Drafts, 37-59. New York: Vintage

Giles, A.E. 1901. Menstruation and Its Disorders. London: Baillière, Tindall and Cox.

Gilman, Sander L., Helen King, Roy Porter, G. S. Rousseau, and Elaine Showalter. 1993. Hysteria Beyond Freud. Berkeley, CA: University of California Press.

Greene, Raymond, and Katharina Dalton. 1953. "The Premenstrual Syndrome." British Medical Journal 1 (4818): 1007-13. https://doi.org/10.1136/bmj.l. 4818.1007.

Halbreich, Uriel. 2007. "The Diagnosis of PMS/PMDD_The Current Debate." In The Premenstrual Syndromes: PMS and PMDD., edited by Patrick Michael Shaughan O'Brien, Andrea J. Rapkin, and Peter J. Schmidt, 9-19. Boca Raton, FL: CRC Press.

Halbreich, Uriel, Jean Endicott, S. Schacht, and J. Nee. 1982. "The Diversity of Premenstrual Changes as Reflected in the Premenstrual Assessment Form." Acta Psychiatrica Scandinavica. https://doi.org/10.1111/j.1600-0447.1982. tb00820.x.

Hartlage, S. Ann, Sally Freels, Nathan Gotman, and Kimberly Yonkers. 2012. "Criteria for Premenstrual Dysphoric Disorder." Archives of General Psychiatry 69 (3): 300. https://doi.org/10.1001/archgenpsychiatry.2011.1368.

Hoffmann, Diane E., and Anita J. Tarzian. 2001. "The Girl Who Cried Pain: A Bias Against Women in the Treatment of Pain." Journal of Law, Medicine and Ethics, no. 29: 13-27. https://doi.org/10.1111/j.1748-720X.2001.tb00037.x.

Hollick, Frederick. 1860. The Marriage Guide or Natural History of Generation; A Private Instructor for Married Persons and Those about to Marry, Both Male and Female. 200th ed. New York: T. W. Strong.

Jing, Zheng, Xunzhe Yang, Khaled MK Ismail, Xiao Y. Chen, and Taixiang Wu. 2009. "Chinese Herbal Medicine for Premenstrual Syndrome." Cochrane Database of Systematic Reviews, January. https://doi.org/10.1002/14651858.CD006414.pub2.

King, Helen. 1998. Hippocrates' Woman: Reading the Female Body in Ancient Greece. London: Routledge.

Knaapen, Loes, and George Weisz. 2008. "The Biomedical Standardization of Premenstrual Syndrome." Studies in History and Philosophy of Science 39 (1): 120-34. https://doi.org/10.1016/j.shpsc.2007.12.009.

Kuczmierczyk, Andrzej R., Anthony H. Labrum, and Carolyn C. Johnson. 1992. "Perception of Family and Work Environments in Women with Premenstrual Syndrome." Journal of Psychosomatic Research 36 (8): 787-95. https://doi. org/10.1016/0022-3999(92)90137-Q. 
Kulkarni, Jayashri. 2013. "PMS Is Real and Denying Its Existence Harms Women." The Conversation. https://theconversation.com/pms-is-real-anddenying-its-existence-harms-women-11714.

Laws, Sophie, Valerie Hey, and Andrea Eagan. 1985. Seeing Red; the Politics of Pre-Menstrual Tension. London: Hutchinson.

Letson, Sue, and Christine P. Dancey. 1996. "Nurses' Perceptions of Irritable Bowel Syndrome (IBS) and Sufferers of IBS.” Journal of Advanced Nursing 23 (5): 96974. https://doi.org/10.1046/j.1365-2648.1996.10416.x.

Lopez, Laureen M., Adrian A. Kaptein, and Frans M. Helmerhorst. 2012. "Oral Contraceptives Containing Drospirenone for Premenstrual Syndrome." Cochrane Database of Systematic Reviews, February. https://doi.org/10.1002/14651858. CD006586.pub4.

Maharaj, Shalini, and Kenneth Trevino. 2015. "A Comprehensive Review of Treatment Options for Premenstrual Syndrome and Premenstrual Dysphoric Disorder." Journal of Psychiatric Practice 21 (5): 334-50. https://doi. org/10.1097/PRA.0000000000000099.

Marjoribanks, Jane, Julie Brown, Patrick Michael Shaughn O’Brien, and Katrina Wyatt. 2013. "Selective Serotonin Reuptake Inhibitors for Premenstrual Syndrome." In Cochrane Database of Systematic Reviews, edited by Julie Brown. Chichester, UK: Wiley. https://doi.org/10.1002/14651858.CD001396.pub3.

Martin, Emily. 1987. The Woman in the Body: A Cultural Analysis of Reproduction. Milton Keynes: Open University Press. https://doi.org/10.1080/14616 700220145650 .

Marván, Ma Luisa, and Claudia Escobedo. 1999. "Premenstrual Symptomatology: Role of Prior Knowledge about Premenstrual Syndrome." Psychosomatic Medicine 61 (2): 163-67. https://doi.org/10.1097/00006842-199903000-00007.

Moos, Rudolf H. 1968. "The Development of a Menstrual Distress Questionnaire." Psychosomatic Medicine 30 (6): 853-67. https://doi.org/10.1097/00006842196811000-00006.

Naheed, Bushra, Jan Herman Kuiper, Olalekan A. Uthman, Fidelma O'Mahony, and Patrick Michael Shaughn O'Brien. 2017. "Non-Contraceptive OestrogenContaining Preparations for Controlling Symptoms of Premenstrual Syndrome." The Cochrane Database of Systematic Reviews 3 (March). https://doi.org/ 10.1002/14651858.CD010503.pub2.

O'Brien, Patrick Michael Shaughn. 2007. "Preface." In The Premenstrual Syndromes: PMS and PMDD, edited by Patrick Michael Shaughan O'Brien, Andrea J Rapkin, and Peter J Schmidt, xi-xii. Boca Raton, FL: CRC Press.

O'Brien, Patrick Michael Shaughn, and Khaled M. K. Ismail. 2007. "History of the Premenstrual Disorders." In The Premenstrual Syndromes: PMS and PMDD, edited by Patrick Michael Shaughan O'Brien, Andrea J. Rapkin, and Peter J. Schmidt, 1-8. Boca Raton, FL, USA: CRC Press.

Quirke, Stephen. 2002. "Manuscript for the Health of Mother and Child." Trans. The Kahun Gynaecological Papyrus. 2002. http://www.ucl.ac.uk/museums-static/digitalegypt/med/birthpapyrus.html.

RCOG. 2016. "Management of Premenstrual Syndrome: Green-Top Guideline No. 48." BJOG: An International Journal of Obstetrics \& Gynaecology 124 (3): 73-105. https://doi.org/10.1111/1471-0528.14260. 
Romans, Sarah, Rose Clarkson, Gillian Einstein, Michele Petrovic, and Donna Stewart. 2012. "Mood and the Menstrual Cycle: A Review of Prospective Data Studies." GENM 9: 361-84. https://doi.org/10.1016/j.genm.2012.07.003.

Simon, Chantal, Hazel Everitt, Francoise Van Dorp, and Matthew Burke. 2014. Oxford Handbook of General Practice. Oxford: Oxford University Press.

Smorgick, Noam, Courtney A. Marsh, Sawsan As-Sanie, Yolanda R. Smith, and Elisabeth H. Quint. 2013. "Prevalence of Pain Syndromes, Mood Conditions, and Asthma in Adolescents and Young Women with Endometriosis." Journal of Pediatric and Adolescent Gynecology 26 (3): 171-75. https://doi.org/10.1016/j. jpag.2012.12.006.

Sonntag, Barbara, and Michael Ludwig. 2012. "An Integrated View on the Luteal Phase: Diagnosis and Treatment in Subfertility." Clinical Endocrinology 77 (4): 500-507. https://doi.org/10.1111/j.1365-2265.2012.04464.x.

Steiner, Meir, M. Macdougall, and E. Brown. 2003. "The Premenstrual Symptoms Screening Tool (PSST) for Clinicians." Archives of Women's Mental Health 6 (3): 203-9. https://doi.org/10.1007/s00737-003-0018-4.

Steiner, Meir, Miki Peer, Mary MacDougall, and Roger Haskett. 2011. "The Premenstrual Tension Syndrome Rating Scales: An Updated Version.” Journal of Affective Disorders 135 (1-3): 82-88. https://doi.org/10.1016/j. jad.2011.06.058.

Ussher, Jane M. 1989. The Psychology of the Female Body. London and New York: Routledge.

- 2003. "The Ongoing Silencing of Women in Families: An Analysis and Rethinking of Premenstrual Syndrome and Therapy." Journal of Family Therapy 25: 388-405. https://doi.org/10.1111/1467-6427.00257.

- 2005. Managing the Monstrous Feminine: Regulating the Reproductive Body. London and New York: Routledge. https://doi.org/10.4324/9780203328422.

- 2011. The Madness of Women: Myth and Experience. London and New York: Routledge. https://doi.org/10.4324/9780203806579.

Ussher, Jane M, Janette Perz, and Julie Mooney-Somers. 2007. "The Experience and Positioning of Affect in the Context of Intersubjectivity: The Case of Premenstrual Syndrome." International Journal of Critical Psychology 21: 144-65.

Veith, Ilza. 1965. Hysteria; The History of a Disease. Chicago: University of Chicago Press.

Walker, Anne E. 1997. "Premenstrual Syndrome.” In The Menstrual Cycle, 144-90. London and New York: Routledge.

Wright, Almroth. 1912. "Militant Hysteria.” Spectator Magazine, March 30, 1912.

Wu, Liping, Zhong He, Hong Zhao, Di Ma, Sijia Zhang, Hanyu Deng, and Tao Liang. 2013. "Chinese Version of Daily Record of Severity of Problems: Reliability and Validity." Journal of Advanced Nursing 69 (2): 449-56. https://doi. org/10.1111/j.1365-2648.2012.06070.x. 
Open Access This chapter is licensed under the terms of the Creative Commons Attribution 4.0 International License (http://creativecommons.org/licenses/ by $/ 4.0 /)$, which permits use, sharing, adaptation, distribution and reproduction in any medium or format, as long as you give appropriate credit to the original author(s) and the source, provide a link to the Creative Commons license and indicate if changes were made.

The images or other third party material in this chapter are included in the chapter's Creative Commons license, unless indicated otherwise in a credit line to the material. If material is not included in the chapter's Creative Commons license and your intended use is not permitted by statutory regulation or exceeds the permitted use, you will need to obtain permission directly from the copyright holder. 